\title{
Zur Frage der Eiweißresorption.
}

\author{
Von
}

Otto Cohnheim und F. Makita (Heidelberg).

(Der Redaktion zugegangen am 7. Juli 1909.)

Der eine von uns hat im vorigen Jahre Untersuchungen über die Resorption von Pepton durch isolierte überlebende Fischdärme angestellt. ${ }^{1}$ ) Die Dünndärme wurden in Ring ersche Lösung gelegt und mit Pepton gefüllt. Nach einigen Stunden konnte in der Außenflüssigkeit eine große Menge Stickstoff nachgewiesen werden, der also durch die Darmwand durchgegangen war, und von diesem Stickstoff war ein beträchtlicher Teil Ammoniak. Zu den Versuchen diente Pepton, das durch Pepsinverdauung von Casein gewonnen war, und außerdem die natürliche Nahrung der Fische. Auch bei der Resorption von Asparaginsäure und Lysin konnte Ammoniak nachgewiesen werden.

Wir haben nun die Versuche wieder aufgenommen, mit der Absicht, die Resorption von solchen Aminosäuren zu studieren, deren Umwandlungsprodukte leicht kenntlich sind. Wir wählten Tyrosin und Glykokoll. Alle in Betracht kommenden Umwandlungsprodukte des Tyrosins mußten die Millonsche, und falls diese in der chlornatriumhaltigen Flüssigkeit nicht anzustellen war, die Paulysche Reaktion ${ }^{2}$ ) geben, eine Rotfärbung mit Diazobenzolsulfosäure und Soda. Aus dem Glykokoll konnte nach der Vermutung von Neubauer ${ }^{3}$ ) Glyoxylsäure entstehen, die durch die umgekehrte Hopkinssche Reaktion, elne Färbung mit Witte-Pepton und konzentrierter Schwefelsăure, leicht nachweisbar ist. ${ }^{4}$ ) Das Tyrosin war teils von

1) 0. Cohnheim, Diese Zeitschrift, Bd. LIX, S. 239 (1909).

2) H. Pauly, Diese Zeitschrift, Bd. XLII, S. 508 (1904).

3) 0. Neubauer, Deutsch. Arch. f. klin. Med., Bd.XCV, S. 211 (1909).

4) H. Eppinger, Hofmeisters Beitr., Bd. VI, S. 492 (1905). E. Granström, ibid., Bd. XI, S. 132 (1908).

Hoppe-Seyler's Zeitschrift f. physiol. Chemie. LXI. 
Merck bezogen, teils hatten wir es uns durch Verdauung von Casein mit Pepsin und Erepsin selbst dargestellt, und das Präparat gereinigt. Das Glykokoll stammte von Kahlbaum.

Die Versuche wurden im April 1909 in dem Laboratorium des Musée océanographique in Monaco angestellt. Wir danken dem Direktor des Instituts, Herrn Professor Richard, und dem Leiter der chemischen Abteilung, Herrn Dr. Ochsner, herzlichst für ihre gütige Unterstützung.

Als Versuchstiere dienten wieder Labriden, Labrus festivus und Grenilabrus pavo. Leider waren sie nicht in genügender Anzahl aufzutreiben, sodaß wir uns genötigt sahen, daneben auch einen anderen, bei Monaco sehr häufigen Fisch, Sargus annularis, zu verwenden, dessen Darm dünn und relativ lang ist. Indessen war die absolute Menge des Resorbierten bei diesen Tieren auffallend geringer als bei den Labriden. Die Därme bewegen sich auch in der Ringerschen Lösung nicht, während dies die Labrus- und Crenilabrusdärme stets tun. Die Zusammensetzung der Ringerschen Lösung und die ganze Versuchsanordnung war die gleiche, wie bei den früheren Versuchen des einen von uns; nur die Versuchsdauer haben wir auf 4-6 Stunden beschränkt.

Unsere sämtlichen Reagenzien, die Lösungen von Glykokoll und Tyrosin, gaben bei der Wasserdampfdestillation mit $\mathrm{BaCO}_{3}$ kein Ammoniak ab. Der Titer einer vorgelegten Oxalsäurelösung änderte sich nicht. Nur mußte die Säurelösung nach beendigter Destillation in Gefäßen aus Jenaer Glas gekocht werden.

\section{Versuche mit Glykokoll.}

4 Därme von Labriden. Außen Ringersche Lösung. Innen $15 \mathrm{ccm}$ Wasser mit $0,25 \mathrm{~g}$ Glykokoll. $5^{1 / 4}$ Stunden. Die ganze Menge der Außenflüssigkeit wird mit Magnesiumcarbonat durch Einleiten von Wasserdampf destilliert, $20 \mathrm{ccm}$ 1/10-n-Oxalsäure werden abgesättigt.

Bei den folgenden Versuchen wird die Destillation mit Baryumcarbonat vorgenommen.

$3^{1 / 2}$ Därme von Crenilabrus pavo, alles kleine Tiere. 
Innen Glykokolllösung von der gleichen Konzentration, außen Ringersche Lösung, der das Blut der betreffenden Fische zugefügt war. Dauer $3^{1 / 2}$ Stunden. Die Außenflüssigkeit wird mit Essigsäure und viel Kochsalz gekocht, das Eiweiß abfiltriert. Das nicht ganz klare Filtrat enthält $16 \mathrm{mg} \mathrm{N}$ und $2 \mathrm{mg} \mathrm{NH}-\mathrm{N}$. Die Flüssigkeit reduziert Silber- und Kupferoxyd nicht und gibt mit Witte-Pepton und konzentrierter Schwefelsäure keine Buntfärbung, enthält also keine Glyoxylsäure.

4 Därme von Labrus und Crenilabrus, 2 von Sargus. Innen Glykokollösung von gleicher Konzentration wie oben, außen Ring ersche Lösung ohne Blut. Die Flüssigkeit enthält $55 \mathrm{mg} \mathrm{N}$, $14 \mathrm{mg} \mathrm{NH}-\mathrm{N}$. Sie reduziert Silber- und Kupferoxyd nicht, gibt keine Hopkinssche Reaktion mit Schwefelsäure und enthält außer Kohlensäure keine flüchtigen Säuren.

4 Sargusdärme und $3^{1 / 2}$ Labrusdärme. Innen $20 \mathrm{ccm}$ Wasser mit $0,4 \mathrm{~g}$ Glykokoll. Außen bluthaltige Ringersche Lösung. 5 Stunden. Der aus der Ringerschen Lösung abströmende Sauerstoff wird durch Natronlauge und Oxalsäure geleitet. Es entweicht kein Ammoniak, wohl aber etwas Kohlensäure. Außenflüssigkeit wird mit Kochsalz und etwas Phosphorsäure gekocht. Sie enthält $48 \mathrm{mg} \mathrm{N}$ und $6 \mathrm{mg} \mathrm{NH} \mathrm{NH}_{3}-\mathrm{N}$. Sie gibt keine Hopkinssche Reaktion.

Auch bei der Resorption von Glykokoll geht also Ammoniak oder, richtiger gesagt, eine flüchtige Base in die Außenflüssigkeit über, aber relativ wenig. Glyoxylsäure wird nicht gebildet. Was für eine Säure auftritt, die das Ammoniak neutralisiert, konnte bei den geringen Mengen, um die es sich handelte, nicht festgestellt werden.

\section{Versuche mit Tyrosin.}

Das Tyrosin wurde in ganz verdünntem $\mathrm{NaHCO}_{3}$ aufgeschwemmt, erwärmt, zur Abstumpfung der Alkalescenz Kohlensäure durchgeleitet, und abkühlen gelassen. Es löst sich relativ wenig Tyrosin, doch werden die ungelösten Krystalle mit in die Därme eingespritzt.

3 Därme von Sargus annularis. Dauer 51/2 Stunden. Die Außenflüssigkeit ist Ring ersche Lösung. Sie enthält $11 \mathrm{mg} \mathrm{N}$, 
$5 \mathrm{mg} \mathrm{NH}-\mathrm{N}$. Mit Diazobenzolsulfosäure und Soda Rotfärbung. Die Millonsche Reaktion ist, wie wir uns überzeugten, in Ringerscher Lösung von der für die Fische erforderlichen Konzentrationen mit Tyrosin und Pepton nicht auszuführen. Phenylhydrazin und Salzsäure gibt auch beim Erwärmen und nach langem Stehen keinen Niederschlag, Silber- und Kupferoxyd werden nicht reduziert.

6 Därme von Sargus annularis. Außen Ringersche Lösung. Dauer $4^{1} / 2$ Stunden. $21 \mathrm{mg} \mathrm{N}, 8 \mathrm{mg} \mathrm{NH}_{3}-\mathrm{N}$. Diazoreaktion positiv. Keine Reduktion von Fehlingscher Lösung, kein Niederschlag mit Phenylhydrazin und Salzsäure.

6 Därme von Labrus festivus, alles kleine Tiere. Außen Ringersche Lösung. Dauer 5-6 Stunden. Außenflüssigkeit ca. $165 \mathrm{ccm}$.

$40 \mathrm{ccm}$, nach Kjeldahl verascht, sättigen ab $2 \mathrm{ccm}{ }^{1 / 10-n-S a ̈ u r e ~}$

40 , mit $\mathrm{BaCO}_{3}$ destilliert,

Die Lösung enthält $11,5 \mathrm{mg} \mathrm{N}$, diese ausschließlich in Form von Ammoniak. Diazoreaktion positiv, keine Reduktion von Fehlingscher Lösung. Kein Niederschlag mit Phenylhydrazin und Salzsäure.

4 Därme von Labrus und Crenilabrus, alles kleine Tiere, sodaß in jeden Darm höchstens $1 \mathrm{ccm}$ der Tyrosinemulsion eingespritzt werden konnte. Außen Ringersche Lösung. 5 $51 / 2$ Stunden. Außenflüssigkeit $200 \mathrm{ccm}$.

$40 \mathrm{ccm}$, nach Kjeldahl verascht, sättigen ab $0,8 \mathrm{ccm} \mathrm{1/10-n-Säure}$

40 , mit $\mathrm{BaCO}_{2}$ destilliert,

Es sind also 5,6 $\mathrm{mg} \mathrm{N}$ vorhanden, diese aber ganz in Form einer flüchtigen Base. Diazoreaktion positiv. Keine Reduktion.

Von den 3 letzten Versuchen wurde die nicht für die Destillation, die Kjeldahlbestimmungen und die Reaktionen verbrauchte Außenflüssigkeit vorsichtig eingeengt, angesäuert und mit vielen Portionen Äther geschüttelt. In den Äther ging nichts über, das die Millonsche oder die Diazoreaktion gab. Der Rückstand gab dagegen eine deutliche Diazoreaktion.

Wir müssen aus diesen Versuchen den Schluß ziehen, daß, wie es früher für das Peptongemenge, für Asparaginsäure 
und Lysin gezeigt worden war, so auch Glykokoll und Tyrosin bei dem Durchtritt durch die resorbierende Darmwand von Fischen desamidiert werden. Für das schwer lösliche Tyrosin läßt sich bei der Wahl der geeigneten Versuchstiere sogar zeigen, daß die Desamidierung eine vollständige ist. Was neben dem Ammoniak für eine Säure gebildet wird, konnten wir infolge der geringen Zahl der zur Verfügung stehenden Fische nicht feststellen, nur erscheint es nach unseren Resultaten nicht wahrscheinlich, daß Ketosäuren auftreten.

Wir haben dann, obwohl von früher schon negative Resultate vorlagen, noch einige Versuche mit den Därmen von Hunden und Katzen gemacht. Die Därme wurden möglichst rasch aus dem Körper entnommen, mit Ringerscher Lösung ausgespült, mit Tyrosin- oder Glykokollösung gefüllt und in körperwarme Ringersche Lösung gelegt, durch die ein Sauerstoffstrom perlte. Nach $1 \frac{1 / 2}{2}$ Stunden wurde die Ringersche Lösung auf Ammoniak, Stickstoff und Diazoreaktion geprüft.

3 junge Hunde von 10 Tagen. Innen Tyrosin.

$420 \mathrm{ccm}$ Außenflüssigkeit, die trüb ist und koagulierbares Eiweiß enthält. Sie enthält $36 \mathrm{mg} \mathrm{N}$, das Filtrat nach Abscheidung des Eiweißes $19 \mathrm{mg} \mathrm{N}$, davon 2,5 mg $\mathrm{NH}_{3}-\mathrm{N}$. Keine Rötung mit Diazobenzolsulfosäure und Soda.

Katze. Innen nur Ringersche Lösung. Außenflüssigkeit $470 \mathrm{ccm}$, trüb, enthält koagulierbares Eiweiß. Sie enthält $2,6 \mathrm{mg} \mathrm{NH}_{3}-\mathrm{N}, 35 \mathrm{mg} \mathrm{N}, 13 \mathrm{mg}$ unkoagulierbaren Stickstoff.

Katze. Innen Glykokoll. Außenflüssigkeit $755 \mathrm{ccm}, 2,7 \mathrm{mg}$ $\mathrm{NH}_{\mathbf{8}}-\mathrm{N}, 41 \mathrm{mg} \mathrm{N}$.

Es geht bei den Säugetierdärmen so viel von den Därmen in Lösung, daß die Versuche nicht anzustellen sind. 